\title{
Der Partizipationsmythos „Partizipation in Reallaboren ist per se transparent und muss es auch sein'6
}

\author{
Alexandra Quint, Sophia Alcántara \& Andreas Seebacher
}

Ein Partizipationsmythos ist ein Komplex von individuellen und kollektiven Vorstellungen über Partizipation, die sich unter den Akteuren eines Reallabors als unhinterfragte Erwartungshaltung hartnäckig halten und-ohne ausreichende Evidenz-Entscheidungen (mit) beeinflussen. Ein solcher Mythos kann entweder bereits bei Beginn der Projekttätigkeit in den Köpfen der Beteiligten vorhanden sein, er kann während der Implementierung entstehen, oder er kann von außen an ein Reallabor herangetragen werden.

Die Vorstellung, dass ein hohes Maß an Transparenz ein notwendiges Kriterium für das Gelingen der gemeinsamen Arbeit darstellt, ist unter ,Reallabor-Akteuren “ weit verbreitet und mit der Erwartungshaltung verknüpft, größtmögliche Transparenz in der Reallaborarbeit zu gewährleisten. Dieser Anspruch lässt sich u. a. darauf zurückführen, dass Transparenz ein normativer, demokratietheoretisch begründbarer und heute allgegenwärtiger Anspruch an Partizipationsprozesse ist. Sie gilt als Kernbestandteil einer Good Governance, als demokratiefördernd und gehört zum heutigen Zeitgeist. Transparenz ist als Qualitätskriterium für (politische) Entscheidungsprozesse und Partizipationsverfahren (zumindest theoretisch) etabliert (zu Qualitätskriterien vgl. Eckart et al. 2018). Die wachsende Skepsis der Bevölkerung gegenüber staatlichen Institutionen und der Zweifel daran, ob Entwicklungen im Sinne des Gemeinwohls vorangetrieben werden (Quint und Parodi 2017), haben diese Entwicklung beflügelt. Entscheidungsträger haben auf diesen Umstand entsprechend reagiert und ein Mehr an Transparenz in unterschiedlichen Prozessen konzeptionell verankert (vgl. Deutscher Städtetag 2013, S. 5).

Erfahrungen aus der Reallaborarbeit machen jedoch deutlich, dass Transparenz in Partizipationsprozessen einer genaueren Betrachtung bedarf, weil ein hohes Maß an Transparenz nicht automatisch zu einem gelingenden Prozess führt. Wird zum Beispiel in einer Arbeitsgruppe an einem Realexperiment gearbeitet, werden dabei Meinungen ausgetauscht und schließlich (Zwischen-)Ergebnisse im Sinne der Transparenz an weitere Akteure innerhalb des Reallabors in einer Präsentation 
oder einem Protokoll weitergegeben oder im Rahmen der Öffentlichkeitsarbeit sogar an Akteure außerhalb des Reallabors weitergegeben, ergibt sich bei später ggf. notwendigem Umlenken die Problematik, dass einmal geäußerte Meinungen oder eingenommene Positionen weniger leicht wieder revidiert werden (können).

Unstrittig ist, dass Transparenz ein gewichtiges Prinzip gesunder Demokratien ist. Sie bildet die Basis für eine erleichterte Dialogführung, eine freie Willensbildung sowie die Förderung der Offenheit gegenüber Neuem. Strittig sind hingegen das präzise Verständnis und die konkrete Umsetzung von Transparenz, das heißt, wo Transparenz anfängt, wie weitreichend und umfassend sie sein sollte und wie viel davon in Partizipationsprozessen tatsächlich förderlich ist. Vertraulichkeit und Transparenz können laut Wewer (2014) als zwei Enden eines Kontinuums gesehen werden. Vertraulichkeit ist dabei so zu verstehen, dass bestimmte Informationen zu Abläufen, Vorhaben, Sachverhalten und Entscheidungen geschützt und nur einem vorab definierten Personenkreis zur Verfügung gestellt werden sollten. Dem gegenüber steht der Transparenzanspruch, der für die möglichst umfassende Offenlegung, Sichtbarmachung und Kommunikation von Informationen steht. Entsprechend dieses Kontinuums gestaltet sich die Umsetzung von Transparenz im tatsächlichen Prozess sehr vielfältig (vgl. Wewer 2014, S. $71 \mathrm{ff}$.). Übertragen auf Reallabore stellen sich mehrere Fragen: Wie viel Transparenz (und worüber) benötigt ein gelingender Partizipationsprozess? Entscheiden die Wissenschaftler(innen) und Initiator(inn)en von Reallaboren (über Fragen der Transparenz) alleine oder - dem Co-Design-Gedanken folgend - gemeinsam mit (welchen) Praxispartnern? Wollen Praxisakteure überhaupt Transparenz und, wenn ja, in welchem Umfang? Welche Prozesse sind überhaupt geeignet, (für wen) transparent gemacht zu werden? Wie kann man mit der potentiell konfliktiven Situation umgehen, die zwischen der in der gesellschaftspolitischen Sphäre gegebenen Transparenzforderung und dem Schutz wissenschaftlicher Ideen und Erkenntnisse oder qua Gesetz zu schützender Daten besteht? Sind Reallabor-Prozesse, in denen nicht alle Akteure über alle Sachverhalte bis ins Detail informiert werden, automatisch schlechte Prozesse? Kann zu viel Transparenz nicht auch überfordern (vgl. hierzu auch den Partizipationsmythos von Seebacher et al. 2018), überfrachten, von zentralen Punkten ablenken?

Um Antworten auf diese Fragen zu finden, kann es hilfreich sein, sich unterschiedliche Arten von Transparenz vor Augen zu führen. Alcántara et al. (2016) unterscheiden im Kontext von Bürger(innen)beteiligung zwischen Transparenz nach innen und nach außen. Angewendet auf Reallabore steht dabei die Transparenz nach außen beispielsweise für den Vorgang der Information der nicht aktiv an der Reallaborarbeit beteiligten Öffentlichkeit über den Prozess, die Teilnehmenden und die Ergebnisse, während die Transparenz nach innen die Information der an der Reallaborarbeit beteiligten Akteure beispielsweise über laufende oder bereits gelaufene Entscheidungsfindungsprozesse innerhalb des 
Reallabors, über die Ergebnisverwertung im Anschluss an ein Verfahren oder über ihre Rolle im gesamten Prozess meint (vgl. Alcántara et al. 2016, S. 51ff.; Heald 2006, S. 29ff.).

Für Reallabore ist Transparenz nach innen in Partizipationsprozessen von großer Bedeutung, insbesondere im Hinblick auf die im Modus transdisziplinärer Forschung anvisierte Zusammenarbeit auf Augenhöhe und die damit verbundene Vertrauensbildung. Das partizipative Gestalten von Transformationsprozessen im Allgemeinen und von Realexperimenten im Besonderen sowie das damit verbundene Integrieren unterschiedlicher Wissens- und Wertebestände erfordert in vehementer Weise den offenen Austausch aller Beteiligten im gemeinsamen Wirken. Das hohe Maß an Engagement, das die Beteiligten in Reallaborprozesse zumeist einbringen (müssen), kann nur durch motivierende Ein- und Ausblicke, eine konsistente und permanente Kommunikation sowie gegenseitiges Vertrauen erzeugt werden. Schäpke et al. (2017, S. 18) plädieren für eine möglichst frühzeitig einsetzende und transparente Vorgehensweise bei der Konzeption von Realexperimenten, damit bereits zu Beginn der gemeinsamen Arbeit eine Abstimmung erfolgen kann zu Forschungsrisiken und Implikationen des Eingreifens in gesellschaftliche Gefüge. Sie stellen Transparenz zudem als Ansatzpunkt zur Handhabe von Konfliktsituationen im Allgemeinen heraus: „Transparenz, gemeinsame Entwicklung von Zielsetzungen und Vorgehensweisen ebenso wie eine (selbst-) reflexive Vorgehensweise der beteiligten Forschenden [...] sind erste Ansatzpunkte zum Umgang mit Konflikten“ (ebd., S. 24). Transparenz nach innen bezieht sich auf das Binnenverhältnis der Akteure in einem Reallabor und fördert die konstruktive Zusammenarbeit. Die hohe Bedeutung, die der Transparenz nach innen zukommt, macht es umso dringlicher, sich in einem Reallabor mit den oben genannten Fragen zu befassen. Dabei sollte zum einen nicht übersehen werden, dass sich Transparenz weder automatisch einstellt noch ein für allemal vereinbart werden kann, sondern der dauernden Reflexion und Verhandlung entlang dieser Fragen bedarf. Zum anderen wäre es verfehlt, Transparenz als etwas Binäres zu verstehen, das ,da ist oder nicht', oder als etwas, das sich für alle Akteure und Prozesse in einem Reallabor identisch konkretisiert; vielmehr gilt es, Transparenz mit Blick auf die verschiedenen Akteure und die vielfältigen Prozesse und Aktivitäten jeweils spezifisch zu konkretisieren.

Transparenz nach außen ermöglicht es im Kontext von Reallaboren, die Komplexität von Transformationsprozessen für einen breiteren Kreis von Akteuren sichtbar zu machen, Bewusstsein für das und Nähe zum Reallabor zu schaffen und bestenfalls zum Aktiv-werden - zum Partizipieren - zu motivieren. Sie erhöht die Akzeptanz und fördert damit die Legitimierung eines Reallabors als Akteur im ,Projektgebiet' (s. zum Reallabor als Akteur auch Seebacher et al. 2018; Waitz et al. 2018). Dabei ist Transparenz zwar als Prinzip in einem Reallabor zu verankern, darf aber nicht aus Prinzip angewendet werden (vgl. hierzu 
Wewer 2014, S. 71f.). Für die Überwindung von Grenzen und die Entwicklung von Neuem sind, insbesondere im Fall von konfliktiven Situationen, geschützte Räume mit reduzierter Transparenz nach außen wichtig. In solchen Räumen kann Vertrauen nach innen zwischen den an einem Reallabor beteiligten Akteuren aufgebaut werden. Dies ist oftmals eine Bedingung dafür, dass sich Akteure überhaupt auf einen offenen Diskurs einlassen. Dieser Prozess kann durch anerkannte Regelwerke unterstützt werden, wie zum Beispiel eine Verschwiegenheitsvereinbarung (,non-disclosure agreement") oder die sog. Chatham-HausRegel (s. dazu https://www.chathamhouse.org/about/chatham-house-rule, zugegriffen am 11.02.2018).

Schließlich und endlich darf nicht vergessen werden, dass Transparenz nicht ausschließlich auf einer sachlichen Ebene betrachtet werden kann, denn Transparenz ist immer auch eine Frage der Wahrnehmung, da einzelne Handlungen oder Sachverhalte von den einzelnen Akteuren unterschiedlich bewertet werden können. Beteiligte wie Beobachtende können das Maß an Transparenz als unzureichend (zu hoch oder zu gering) einstufen und in Konsequenz sogar den Prozess verlassen. Es ist ein genaues Ermitteln der Anforderung an und der Umsetzung von Transparenz, das den Weg hin zu gelingenden Reallaborprozessen ebnet.

\section{Literatur}

Alcántara, S., Bach, N., Kuhn, R., \& Ullrich, P. (2016). Demokratietheorie und Partizipationspraxis - Analyse und Anwendungspotentiale deliberativer Verfahren. Wiesbaden: Springer VS.

Deutscher Städtetag (2013). Thesen zur Weiterentwicklung lokaler Demokratie. http:// www.netzwerk-buergerbeteiligung.de/fileadmin/Inhalte/PDF-Dokumente/thesenpapier lokale_demokratie_endfassung_ha_07_11_2013.pdf. Zugegriffen am 09.01.2018.

Eckart, J., Ley, A., Häußler, E., \& Erl, Th. (2018). Leitfragen für die Gestaltung von Partizipationsprozessen in Reallaboren. In R. Defila \& A. Di Giulio (Hrsg.), Transdisziplinär und transformativ forschen. Eine Methodensammlung (S. 105-135). Wiesbaden: Springer VS.

Heald, D. (2006). Varieties of Transparency. Proceedings of the British Academy, 135, S. 25-43. http://www.davidheald.com/publications/healdvarieties.pdf. Zugegriffen am 12.01.2018.

Quint, A., \& Parodi, O. (2017). Die Zukunft europäischer Städte - Herausforderungen an eine nachhaltige Entwicklung. Stadt + Raum, 38 (2), (S. 74-76).

Schäpke, N., Stelzer, F., Bergmann, M., Singer-Brodowski, M., Wanner, M., Caniglia, G., \& Lang, D. J. (2017). Reallabore im Kontext transformativer Forschung. Ansatzpunkte zur Konzeption und Einbettung in den internationalen Forschungsstand. (No. 1/2017). Leuphana Universität Lüneburg, Institut für Ethik und Transdisziplinäre Nachhaltigkeitsforschung. 
Seebacher, A., Alcántara, S., \& Quint, A. (2018). Akteure in Reallaboren - Reallabore als Akteure. In R. Defila \& A. Di Giulio (Hrsg.), Transdisziplinär und transformativ forschen. Eine Methodensammlung (S. 155-159). Wiesbaden: Springer VS.

Waitz, C., Quint, A., Trenks, H., Lezuo, D., Jäkel, A., Wäsche, H., \& Parodi, O. (2018). Das Reallabor als Motor für nachhaltige Quartiersentwicklung - Erfahrungen aus dem Karlsruher Experimentierraum. Berichte. Geographie und Landeskunde, 91 (1), (S. 6780). (im Druck).

Wewer, G. (2014). Open Government, Staat und Demokratie. Aufsätze zu Transparenz, Partizipation und Kollaboration. Berlin: edition sigma.

\section{Die zwei weiteren Partizipationsmythen in diesem Buch}

Alcántara, S., Quint, A., \& Seebacher, A. (2018). Der Partizipationsmythos „Partizipation in Reallaboren muss repräsentativ sein“. In R. Defila \& A. Di Giulio (Hrsg.), Transdisziplinär und transformativ forschen. Eine Methodensammlung (S. 137-141). Wiesbaden: Springer VS.

Seebacher, A., Alcántara, S., \& Quint, A. (2018). Der Partizipationsmythos „Partizipation bedeutet, alle immer an allem zu beteiligen“. In R. Defila \& A. Di Giulio (Hrsg.), Transdisziplinär und transformativ forschen. Eine Methodensammlung (S. 101-104). Wiesbaden: Springer VS.

Open Access Dieses Kapitel wird unter der Creative Commons Namensnennung 4.0 International Lizenz (http://creativecommons.org/licenses/by/4.0/deed.de) veröffentlicht, welche die Nutzung, Vervielfältigung, Bearbeitung, Verbreitung und Wiedergabe in jeglichem Medium und Format erlaubt, sofern Sie den/die ursprünglichen Autor(en) und die Quelle ordnungsgemäß nennen, einen Link zur Creative Commons Lizenz beifügen und angeben, ob Änderungen vorgenommen wurden.

Die in diesem Kapitel enthaltenen Bilder und sonstiges Drittmaterial unterliegen ebenfalls der genannten Creative Commons Lizenz, sofern sich aus der Abbildungslegende nichts anderes ergibt. Sofern das betreffende Material nicht unter der genannten Creative Commons Lizenz steht und die betreffende Handlung nicht nach gesetzlichen Vorschriften erlaubt ist, ist für die oben aufgeführten Weiterverwendungen des Materials die Einwilligung des jeweiligen Rechteinhabers einzuholen. 